\title{
Special issue on quantum programming languages
}

\author{
PETER SELINGER
}

Special Issue Editor

Department of Mathematics and Statistics, Dalhousie University, Halifax, Nova Scotia, Canada

Received 15 December 2005

\section{Preface}

This special issue of Mathematical Structures in Computer Science grew out of the 2nd International Workshop on Quantum Programming Languages (QPL 2004), which was held July 12-13, 2004 in Turku, Finland. The purpose of the workshop was to bring together researchers working on mathematical formalisms and programming languages for quantum computing. It was the second in a series of workshops aimed at addressing a growing interest in logical tools, languages, and semantical methods for analysing quantum computation.

The scope of the workshop included the following topics: the syntax and semantics of quantum programming languages; new paradigms for quantum programming; the specification of quantum algorithms; higher-order quantum computation; quantum data types; reversible computation; axiomatic approaches to quantum computation; concurrent and distributed quantum computation; the compilation of quantum programs; semantical methods in quantum information theory; and categorical models for quantum computation.

QPL 2004 was held as an affiliated event of the 19th Annual IEEE Symposium on Logic in Computer Science (LICS 2004), and was also co-located with the 31st International Colloquium on Automata, Languages and Programming (ICALP 2004). The workshop had 34 registered participants, as well as a number of casual participants who dropped in from the co-located conferences. The workshop program consisted of an invited lecture by Richard Jozsa (Bristol), entitled 'On the structure of quantum algorithms and the role of classical mathematics', as well as eleven contributed papers, which were selected by a program committee consisting of Samson Abramsky, Prakash Panangaden and Peter Selinger. The workshop also featured a discussion session, with a vigorious debate on the future of quantum programming language research, its potential applications, and the role of foundational issues.

This issue contains a selection of articles related to the subject of the QPL 2004 workshop. Many of them are the final versions of papers that were first presented at the workshop. I would like to thank the editor-in-chief, Giuseppe Longo, who has handled the refereeing of the paper by Selinger and Valiron. I would also like to take this opportunity 
to thank the 16 anonymous referees who participated in the preparation of this volume, and without whose work the selection of such a fine collection of articles would not have been possible.

Halifax, December 2005

Peter Selinger

(Special issue editor) 